\title{
Pressure-Induced Emission Enhancements and Ripening of Zinc-blende Cadmium Selenide Nanocrystals
}

Pengfei Lv, Songrui Yang, Chuang Liu, Zhiwei Ma, Yaping Chen, Guanjun Xiao* and Bo Zou

State Key Laboratory of Superhard Materials, College of Physics, Jilin University, Changchun 130012, China 


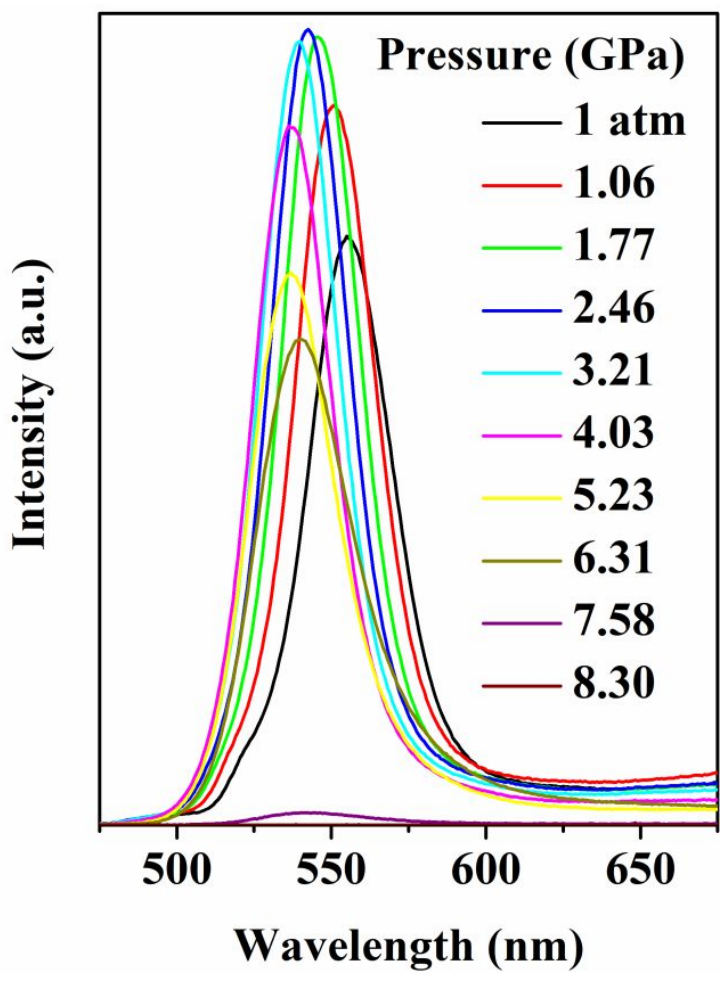

Figure S1. Pressure-dependent PL behaviors of CdSe NCs without stearic acid. 


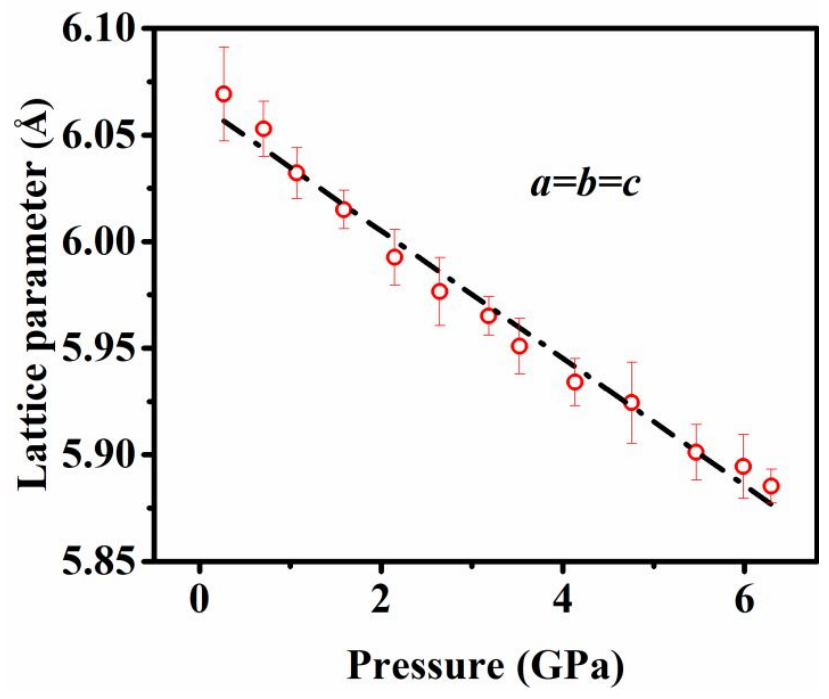

Figure S2. Lattice parameter evolutions of Zb-CdSe NCs under pressure. 

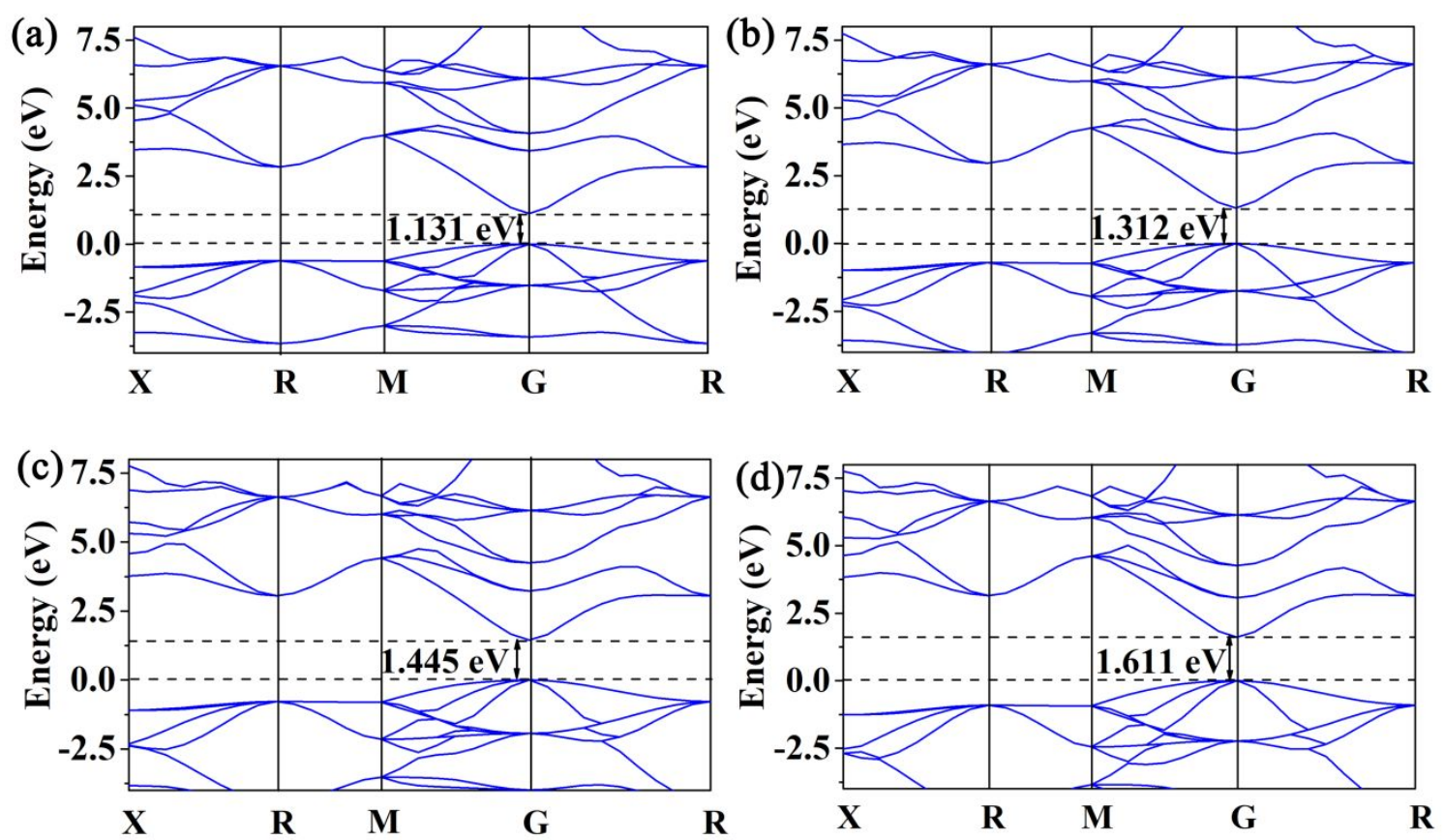

Figure S3. Computational band gaps at $0 \mathrm{GPa}(\mathrm{a}), 4 \mathrm{GPa}(\mathrm{b}), 8 \mathrm{GPa}$ (c) and 15 $\mathrm{GPa}(\mathrm{d})$. 


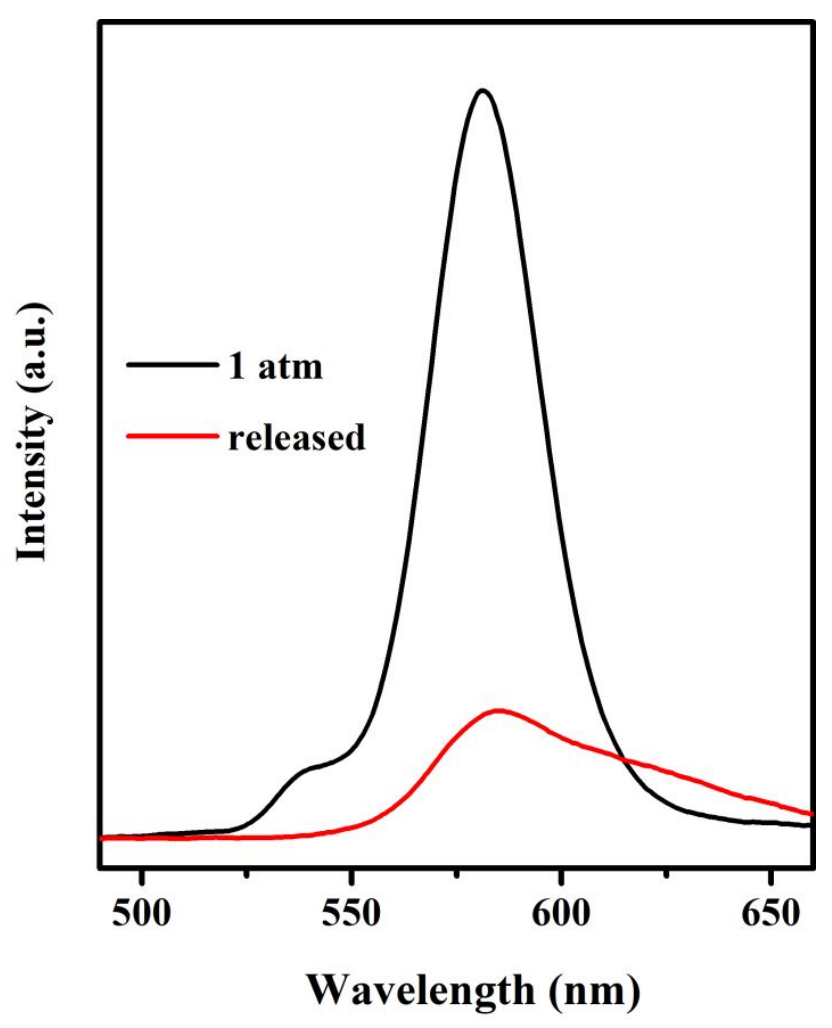

Figure S4. PL spectra of ambient and decompressed Zb-CdSe NCs. 
(a)

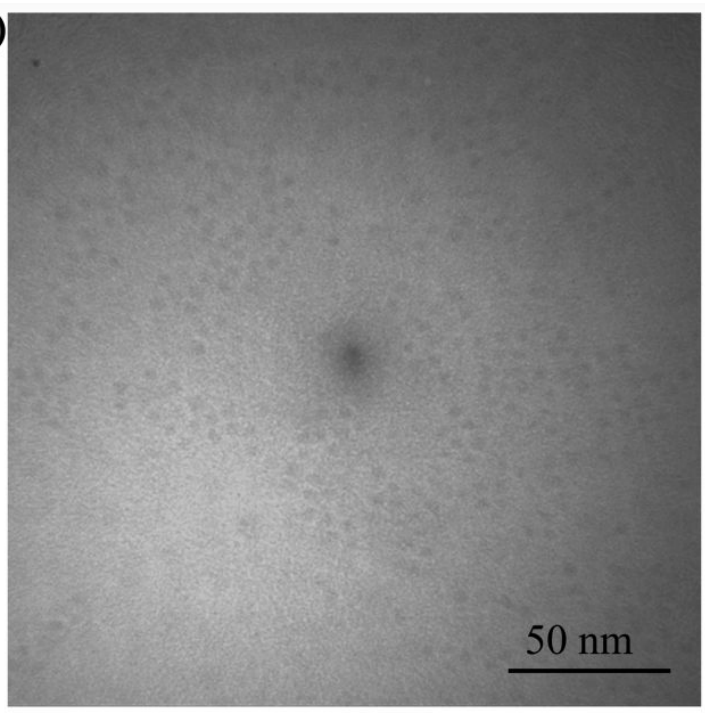

(b)

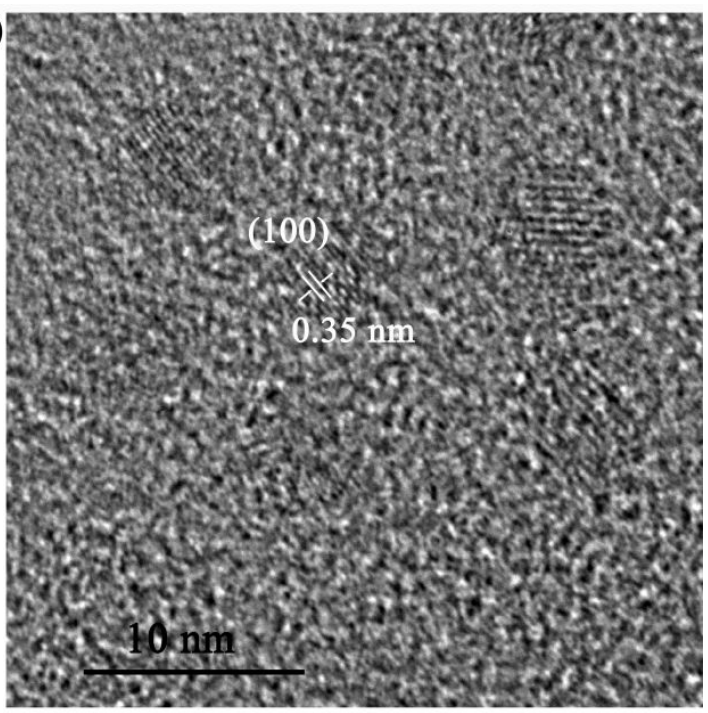

Figure S5. TEM (a) and HRTEM (b) images of the released Zb-CdSe NCs. 


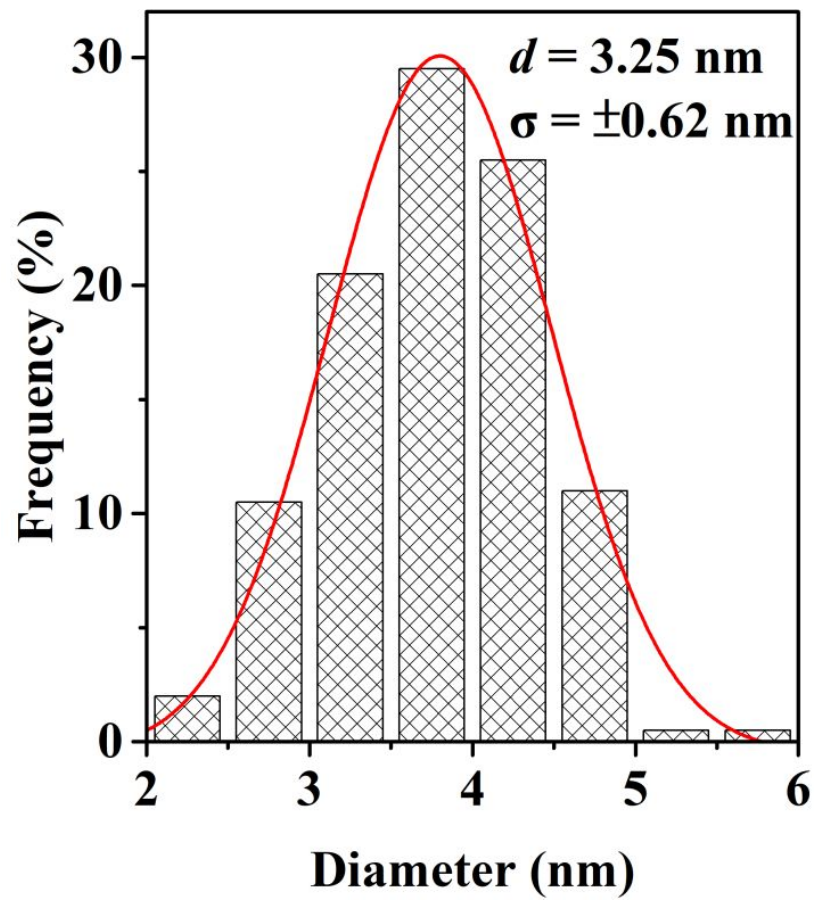

Figure S6. Corresponding size distribution of released Zb-CdSe NCs. 